\title{
Detecting Life and Biology-Related Parameters on Mars
}

\author{
Gilbert V. Levin, Spherix Incorporated, 12051 Indian Creek Court, Beltsville, MD 20705, 301-419-3900; 301-210-4908 (fax); \\ glevin@spherix.com \\ Joseph D. Miller, University of Southern California, Keck School of Medicine, Department of Cell and Neurobiology, 1333 \\ San Pablo Street, BMT 401, Los Angeles, CA 9003384; 323-442-1629; 323-442-3466 (fax); jdm@usc.edu \\ Patricia A. Straat, 830 Windy Knoll, Sykesville, MD 217841; 410-442-1582; 410-992-8429 (fax); pstraat@starpower.net \\ Robert Lodder, A123 ASTeCC Building, University of Kentucky, Lexington, KY, 859-257-9232; FAX; rlodder@uky.edu \\ Richard B. Hoover, NASA/ Marshall Space Flight Center/NSSTC, Huntsville, AL 35805; 256-961-7770; 256-961-7216 (fax); \\ richard.hoover@nasa.gov
}

\begin{abstract}
An integrated, miniaturized, low-power instrument capable of the detection and early characterization of microbial life in the soil of Mars is proposed. Based on the detection and monitoring of on-going metabolism as being the surest evidence for extant life, the experiments will probe for chirality in metabolism, for circadian rhythm, and for photosynthesis. However, the instrument package will also be able to detect biosignatures and a variety of other physical and chemical parameters of the Martian surface that have significance for life. These include the presence and the physical state of water, the existence of an oxidant, the $\mathrm{pH}$ and the penetrability of the soil. Using the legacy of the 1976 Viking Labeled Release (LR) life detection experiment in conjunction with state-of-the-art laser diode spectral analysis, the instrument can be flown stand-alone, with or without a rover, or as part of an MSL-type mission. Sterility for experiment integrity and for planetary protection is provided.
\end{abstract}

Keywords: life on Mars, Viking LR experiment, laser diode analyses, SSSI, life detection experiments, chiral metabolism, photosynthesis, circadian rhythm, water on Mars, Mars soil properties, robotic instruments, spacecraft sterilization

\section{INTRODUCTION AND BACKGROUND}

The search for life on Mars remains one of the foremost scientific issues of our time, proclaimed by NASA as its highest priority. Although the Viking Mission Labeled Release Life Detection Experiment (LR) was positive, indicative of Martian microbial metabolism in accordance with its pre-mission criteria, scientists reacted with caution. The Experimenters initially said only that the results were "consistent with a biological explanation" [1]. By 1986, further study of the data and failure to reproduce the results abiologically in the laboratory prompted the statement, "it is more likely than not that the LR detected life on Mars"[2]. Over the 30 years since Viking, scores of hypotheses have been proposed to explain away the biological interpretation of the LR findings on Mars. They include the failure of the Viking organic analysis experiment (GCMS) to find organic matter, the putative presence of hydrogen peroxide in the Martian soil, UV radiation, ionizing radiation, the thin atmosphere and cold temperatures. All have been found wanting, and none was shown capable of duplicating the Mars LR test and control data (Levin - SPIE 2006). Together with the study of new data from Mars and Earth, this led Levin to conclude (Levin SPIE 1997) that the Viking LR experiment had, in fact, detected living microorganisms in the Martian soil. Currently, the last remaining rationale cited by those believing the Martian surface to be sterile, is the presumed absence of liferequiring liquid water. However, evidence ${ }^{[3],[4],[5],[6],[7]}$ increasingly indicates the presence of liquid water or moisture on the surface of Mars. Although there is a recent trend toward accepting that the Mars LR experiment did, in fact, detect life ${ }^{[8]}$, the general consensus remains negative. The purpose of the herein proposed Mars instrument package is to resolve the issue unambiguously.

\section{KNOWLedGe Gained SinCE VIKING}

Since Viking, NASA and ESA missions to Mars have approached the life issue indirectly. A series of NASA missions has sought to "follow the water" to find potential habitats of extinct or extant life as precursors to seeking life itself. Plans exist to follow these explorations with a "Mars Sample Return Mission" that would acquire material from such selected places and send it to Earth for detailed analysis. Similarly, ESA missions have sought indirect evidence of life, past or present. These spacecraft landing on Mars have made important scientific discoveries related to Mars as a possible habitat. Additional relevant information has been produced by multi-channel observations by Mars orbiters and by Earth-based telescopes.

Methan $^{[9]}$ and formaldehyde ${ }^{[10]}$ have been detected in the Martian atmosphere, both commonly associated with metabolism. Recently, calculations ${ }^{[11]}$ have shown it to be difficult to maintain that the levels of methane detected on Mars are sustained by presumed volcanic activity - even when that activity is projected at levels far above any found, or likely, on Mars. This leaves biological organisms as the most likely, perhaps only, source of this short-lived gas.

In addition to the studies of and on Mars, terrestrial studies in formerly described "extreme environments" and deemed inimical to life have revealed a continuum of life throughout the surface of the Earth, its atmosphere, the lowest depths of its sea, and thousands of meters below sea bottom, including inside metamorphic and even igneous rock found 


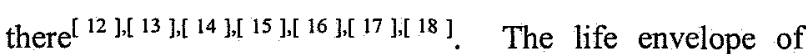
temperature, pressure, $\mathrm{pH}$ and other limiting factors has been greatly expanded.

All of the many findings since Viking have supported, or been consistent with, the presence of extant life on Mars. Equally important, no finding(s) has eliminated that possibility, or made it unlikely. The possibility of a lifeless Mars has been further significantly reduced by experiments $\left[\begin{array}{ll}{[19} & 1\end{array}\right.$ that have shown that terrestrial microorganisms survive the various stresses that would be placed on impact-produced ejecta leaving Earth and eventually landing on Mars.

The last remaining barrier to life cited by those believing the Martian surface to be sterile is the presumed absence of liferequiring liquid water. Both thermodynamic theory and experimental evidence ${ }^{[20],[21],[22],[23],[24]}$, increasingly indicate the presence of liquid water or moisture on the surface of Mars. The existence of liquid water on Mars is also suggested by images of patterned ground with doublerimmed polygons that are analogous to the double-rimmed polygons that form as ice wedges grow by repeated freezethaw of water ice in the permafrost of Earth ${ }^{[25]}$.

The newer findings have induced most of those who have denied the presence of liquid water on the surface of Mars to cite the possibility of liquid water and life in underground "oases." Nonetheless, because of the major implications of the existence of life on Mars, progress toward a change in paradigm, although palpable in recent years, has been slow. New and unambiguous experimental evidence from Mars would be the surest means of settling the issue.

\section{THE EXPERIMENTS}

\section{A. The TWEEL Suite}

The TWEEL suite of experiments is based on the radio carbon labeling technique of the Viking $L R$ experiment

\section{Chirality in Metabolism}

A simple, but highly significant modification of the LR experiment is proposed. It consists of small, fountain pensized, projectiles, "TWEELs," (Twin Wireless Experiment for Extraterrestrial Life) named after fictional Martian birds that dive beak first into the soil ${ }^{[26]}$ each containing duplicate sample chambers. Shot from the lander or rover, a TWEEL lands in uncontaminated soil, upwind from the lander or rover. It plunges into the soil, obtaining its sample from the force of its penetration. Its duplicate samples permit $14 \mathrm{C}$ labeled isomers of optically active substrates to be injected separately onto identical samples of Martian soil. Should ensuing reactions, as determined by evolving radioactive gas, show a preference for either isomer, this would confirm the biological nature of the active agent in the soil. All known forms of life use and make primarily L-amino acids and D-sugars. Rare exceptions can make or use the opposite isomers, but they still rely on a ubiquitous homochirality for their principal life functions. This preference for molecular handedness in a reaction occurs only in living systems, chemical reactions being unable to distinguish between isomers since they are chemically identical. The Viking LR substrates are shown in Table 1. Two of them, alanine and lactate occur as optical isomers. In order to detect any life having isomeric preferences opposite to those of organisms on Earth, both isomers of those compounds were included in the Viking LR experiment. In the proposed TWEEL experiment, the isomers would be separated and tested individually. Organic compounds in addition to those in Table 1 would be injected onto Martian soil samples captured by TWEELs. One would be the sulfur-bearing, life-essential amino acid, cysteine. This seems a good choice since Viking found ${ }^{[27]}$ the surface of Mars to contain some two orders of magnitude more sulfur than the average on Earth.

TWEELs are launched in the morning to take advantage of the rising temperature. Using its integral battery, a TWEEL's temperature is raised to $10^{\circ} \mathrm{C}$ just prior to launch. This ensures liquidity of the nutrient so that it can be dispersed through the incoming soil sample that breaks the ampoule upon impact. The soil sample is then held at $10^{\circ} \mathrm{C}$, the temperature at which the Viking LR samples were maintained, for five sols. except for the increased temperatures required for the temperature entrained circadian rhythm experiments. As in the case with the Viking LR, the radioactive gas in each TWEEL headspace is measured every 4 minutes for the first four hours, to obtain early kinetics, and every 16 minutes thereafter. The data are radioed back to the lander or to an orbiter for relay to Earth. Commands from Earth can change a TWEEL's program should early data give cause.

The Chiral LR experiment can produce results beyond the confirmation of life on Mars. Should a chiral preference be found that is the same as that of Earth life that would constitute a strong indication of a common origin of life on the two planets. The origin could have occurred on either planet, or on a third source. Should a chiral preference opposite to that on Earth be found, this would establish that Earth and Mars life derived from separate sources, greatly increasing the prospects for life on many planets throughout the universe. Should the Martian soil react equally with both isomers, the most likely explanation would be that the active agent is chemical.

\section{Circadian Rhythm}

All known organisms exhibit circadian rhythms (entrained by the Earth's daily cycle of approximately 24 hours) in their metabolism, thus providing an unambiguous biomarker. The molecular substrates of the circadian timekeeping system have been identified in mammals, fruit 
TABLE 1. Labeled Release Nutrients

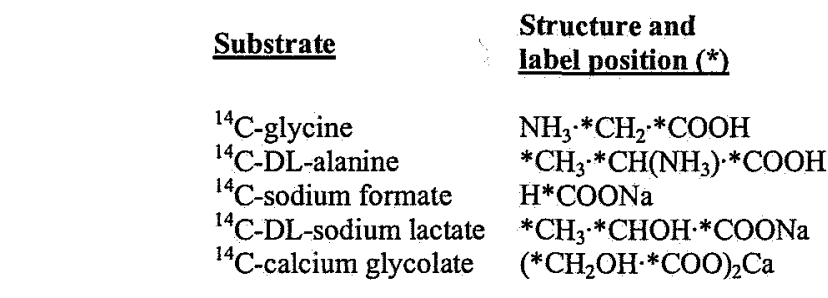

${ }^{*}$ Total $=34 \mathrm{uCi}$, which yields $6.8 \times 10^{7} \mathrm{dpm} \mathrm{ml^{-1 }}$

flies, sea slugs and even Cyanobacteria. While the most potent terrestrial stimulus capable of entraining these rhythms is the $24 \mathrm{hr}$ light/dark cycle, a variety of other agents (temperature, nutrient availability, voluntary locomotor activity) induce entraining effects, although weaker. Evolutionary considerations suggest that an endogenous clock, with a period similar to the rotational period of the planet of origin, may be strongly selected in environments in which life evolves. This, in turn, reflects the importance to the organism of detecting "time of day", e.g., normally diurnal animals awake at night would be at much greater risk of predation; and heliotropism would be of no value to a plant at night. Furthermore, since daylight may be obscured by meteorological or environmental factors (e.g., burrowing animals spend most of the day underground), there appears to have been strong selection pressure for the evolution of an endogenous circadian clock capable of "keeping time" even under such circumstances.

A case has been made ${ }^{[28]}$ that the Viking LR experiment may have exhibited circadian rhythms in the metabolism of microorganisms entrained to a remnant temperature cycle (2 degree $\mathrm{C}$ amplitude), as evidenced by rhythmic oscillations in their evolution of radioactive gas in the lander that, in turn, reflected the ambient daily temperature cycle. However, confirmation and extension of the relatively small database supporting this possibility is desired. TWEELs will be able to determine whether or not the signal from an LR type experiment exhibits circadian rhythmicity, and, if so, to examine its characteristics over time.

After the TWEEL lands and activates, it is maintained at $10^{\circ}$ $\mathrm{C}$, and a five-sol baseline period is obtained in the dark, opaque TWEEL for background. Then a photic stimulus, in the form of bright broad spectrum white light from a miniature LED transmitted through the sample by a light pipe diffuser, will be presented to the sample in one chamber for one hour beginning approximately two hours after local sundown. This potentially-entraining stimulus will be presented each sol for five consecutive sols, but at intervals of $26 \mathrm{hr}$, as opposed to the $24.66 \mathrm{hr}$ Martian daily period. In the duplicate chamber of the TWEEL, the same light stimulus will be presented, but only once every ten hours. After the first five sols, no further light will be applied to either chamber. The ${ }^{14} \mathrm{C}$ gas evolved in the

$\begin{array}{lll}\text { Concentration } & \underline{\mu C i ~ \text { ML }^{-1 *}} & \begin{array}{l}\text { Specific Activity } \\ \text { (Ci/Mole) }\end{array} \\ 2.5 \times 10^{-4} \mathrm{M} & 4 & 16 \\ 5.0 \times 10^{-4} \mathrm{M} & 12 & 48 \\ 2.5 \times 10^{-4} \mathrm{M} & 2 & 8 \\ 5.0 \times 10^{-4} \mathrm{M} & 12 & 48 \\ 2.5 \times 10^{-4} \mathrm{M} & 4 & 16\end{array}$

experiment will then be monitored for an additional five days.

In an analogous experiment we will employ another potent zeitgeber, a temperature pulse. An increase of 5 degrees $\mathrm{C}$ above the ambient will be presented for one hour approximately two hours after local sundown. This stimulus will be given sequentially every $26 \mathrm{hr}$ for five sols, just as in the light pulse experiment. Gas release will be monitored for the rest of the TWEEL cycle.

Since light is the most potent terrestrial entraining stimulus, the expectation is that some sub-population of temperatureentrained microorganisms will "break away" from the temperature zeitgeber and entrain to the daily $26 \mathrm{hr}$ light presentations. Thus a second circadian rhythm should appear in the metabolic record obtained by monitoring the evolved ${ }^{14} \mathrm{C}$ gas. If so, this rhythm should be out of phase with the temperature-entrained rhythm, and should exhibit a period of $26 \mathrm{hr}$, rather than the Martian $24.66 \mathrm{hr}$ natural cycle.

Exactly the same rationale applies to the temperature pulse experiment. If the TWEELs can maintain about a twodegree $\mathrm{C}$ amplitude in the diurnal temperature cycle, the $5^{\circ}$ $\mathrm{C}$ pulse proposed here should be able to entrain a population of putative Martian microbes and produce a second phaseshifted rhythm with a period of $26 \mathrm{hr}$. The control chamber of this TWEEL will receive the $5^{\circ} \mathrm{C}$ temperature pulse every ten hours. Results will be determined by monitoring evolved gas.

Biologically-mediated circadian rhythms should entrain to the $26 \mathrm{hr}$ photic or temperature cycles, but not to the ten $\mathrm{hr}$ cycles. In contrast, if any non-biological mechanism becomes synchronized to the photic stimulus or the thermal stimulus, the period of application should not matter. Tenhour entrainment would be expected in this case. In addition, in the case of biology, the photically-entrained or thermally-entrained rhythms should persist for at least some cycles in the absence of stimulation during the final ten days of the experiment (demonstrating a free run of the newly entrained components).

The observation of entrainment of gas release with a period of $26 \mathrm{hr}$, followed by a free-running rhythm, would constitute excellent evidence for a biological process. 
Furthermore, failure of entrainment to a ten hr zeitgeber cycle would be a precise analog of terrestrial circadian biology.

\section{Photosynthesis}

While the LR has been successful in detecting heterotrophic life, many biologists believe that the first form of life to evolve must have been phototrophic, and that phototrophs must exist on any planet harboring life. Accordingly, a modification of the LR experiment is proposed to assay for activity of photosynthetic organisms present in the sample. A simple experiment is reported in Table 2. To generate the data, a pure culture of Chlorella Pyrenoidosa, a strict phototroph, was exposed to ${ }^{14} \mathrm{CO}_{2}$ in the light, and then subjected to alternate dark and light periods. The production and fixation of ${ }^{14} \mathrm{CO}_{2}$ was monitored throughout the experiment. The results are shown in Figure 1.

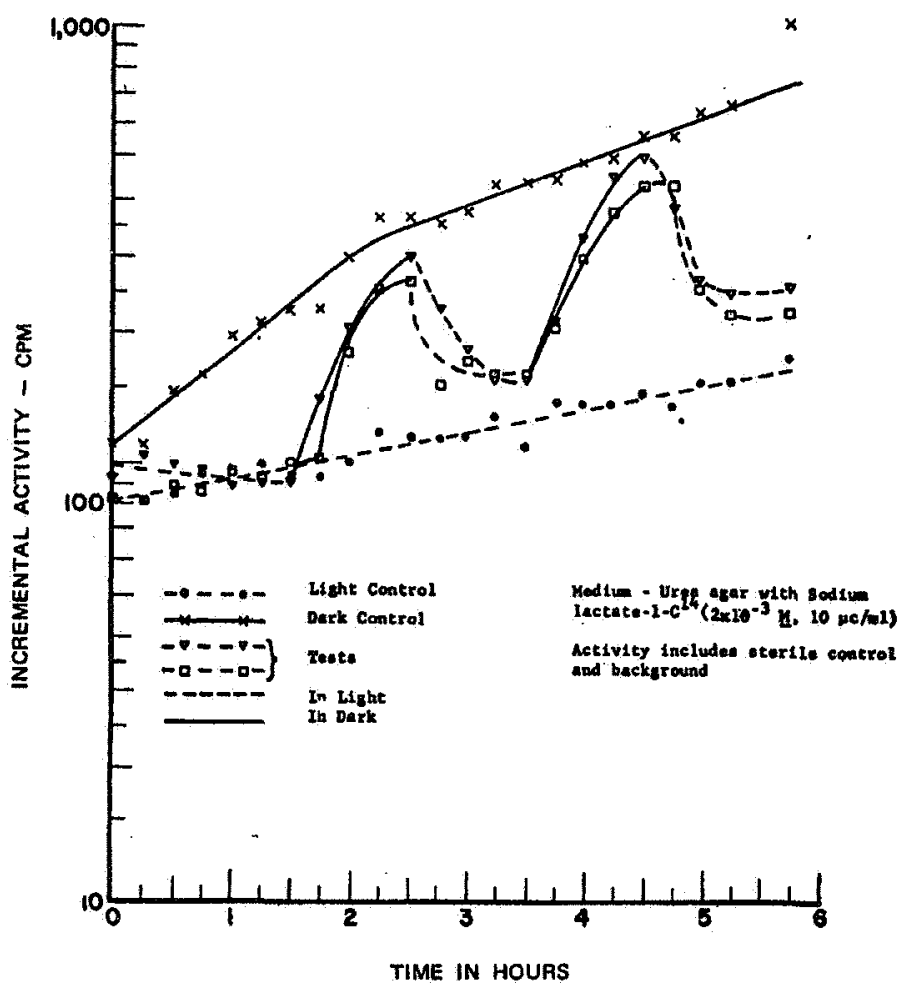

Figure 1. Detection of Photosynthesis of Metabolically Derived ${ }^{14} \mathrm{CO}_{2}$

Reproduced from Levin, G.V., A.H. Heim, M.F. Thompson, D.R. Beem and N.H. Horowtiz, in Life Sciences and Space Research II, p. 130, North-Holland Publishing Co., Amsterdam (1964).

Table 2. Wavelengths of Diodes and Corresponding Chemical Functional Groups.

\begin{tabular}{|l|l|}
\hline \multicolumn{1}{|c|}{ Substance } & \multicolumn{1}{|c|}{ Wavelength(s) } \\
\hline $\begin{array}{l}\text { Residual UV protectant molecules from } \\
\text { biofilms }\end{array}$ & $340 \mathrm{~nm}$ \\
\hline $\begin{array}{l}\text { Residual phototrophic endolithic } \\
\text { biofilms, sulfides }\end{array}$ & $470 \mathrm{~nm}, 530, \mathrm{~nm}, 635 \mathrm{~nm}$ \\
\hline Water, -OH, $=\mathrm{C}=\mathrm{H}$ & $1445 \mathrm{~nm}, 1940 \mathrm{~nm}, 1982 \mathrm{~nm}$ \\
\hline$-\mathrm{CH} 2,-\mathrm{CH} 3$, various -C-H stretches & $\begin{array}{l}1680 \mathrm{~nm}, 1722 \mathrm{~nm}, 1734 \mathrm{~nm}, 1759 \mathrm{~nm}, 1778 \\
\mathrm{~nm}, 1818 \mathrm{~nm}\end{array}$ \\
\hline -NH2, amines, protein amides & $\begin{array}{l}2100 \mathrm{~nm}, 2139 \mathrm{~nm}, 2180 \mathrm{~nm}, 2190 \mathrm{~nm}, 2208 \\
\mathrm{~nm}, 2230 \mathrm{~nm}\end{array}$ \\
\hline Lipids, -CH & $2270 \mathrm{~nm}, 2310 \mathrm{~nm}, 2336 \mathrm{~nm}, 2348 \mathrm{~nm}$ \\
\hline Baseline for reflectance and target angle & $1064 \mathrm{~nm}$ \\
\hline
\end{tabular}


Based on this work, a test for photosynthesis is made possible by including a white LED in each TWEEL, and piping the light to the soil sample. Should any microorganisms be present in the sample and evolve labeled gas, turning the light on and off at two-hour intervals would produce a correlating modulation in the signal amplitude. This experiment would be operated in conjunction with the Circadian Rhythm experiment, they being mutually supportive. If photosynthetic organisms are in the sample, they would absorb some of the carbon gas evolved by heterotrophic metabolism of the substrates in the dark, which the monitoring of the gas in the headspace of the TWEEL would reveal.

\section{Penetrability of Soil}

The nosecone of each TWEEL will contain a miniature penetrometer. The aerodynamic characteristics of the TWEEL will cause essentially vertical entry into the soil. The height to which the TWEEL is programmed by the launch angle and the force of the squib propellant, and the Martian gravity constant permit calibration of the penetrometer to Martian conditions.

\section{Surface Temperature of Soil}

Each TWEEL will be equipped with a thermistor capable of reading over the range of -50 to $+25 \mathrm{deg}$. C. Thermistors will be placed on the ends of anti-submergence flanges so that the temperature of the soil. Time-tagged temperature measurements will be radioed to the spacecraft every hour. The TWEELs will be thermally insulated to conserve energy when being heated.

\section{B. The SSSI Suite}

The Solid State Spectral Imager (SSSI) is a new method to detect living organisms or biological fossils. The SSSI utilizes a Hadamard pulse sequence in a systematic spectroscopic search for unknown biosignatures at a distance, while a CRISP (complementary randomized integrated sensing and processing) pulse sequence enables a rapid remote scan for moisture in all of its forms. In a Walsh-Hadamard sequence, multiple laser diodes illuminate the target at the same time, increasing the number of photons received at the photo detector. The WalshHadamard sequence can be demultiplexed to individual wavelength responses with a matrix-vector multiply ${ }^{[29]}$. Benefits of generating encoding sequences by this method include equivalent numbers of on and off states for each sequence and a constant number of diodes in the on state at each resolution point of a data acquisition period.

The SSSI is composed of three principal components: a laser diode array (LDA), a Collector/Detector Unit (CDU), and a processing unit, as shown in the block diagram in Figure 2. The LDA for the full configuration contains 5 rows of 24 laser diodes, with each row containing all of the wavelengths listed in Table 2 . The processing unit drives the LDA, samples data from the CDU, and classifies the samples using the BEST The Bootstrap Error-adjusted Single-sample Technique (BEST) algorithm.

Laser diodes are positioned within the array so that each laser diode illuminates a different point. When the remote sensing mast on which it is mounted rotates, the spot illuminated by each laser diode in the new position is the same spot previously lit by an adjacent laser diode. Multiple rows of laser diodes are included to reduce the amount of motion needed to complete a scan. An added benefit is that should some laser diodes fail, SSSI can compensate by using laser diodes from a different row to cover for the failed laser diodes. Though scanning speed would be reduced, SSSI would still be able to collect data using all of its wavelengths. SSSI can operate either during day or night. SSSI does not rely on sunlight to illuminate targets, however, and reduced background light at night will reduce the signal-to-noise ratio.

The amount of time required to complete a scan and the amount of data generated depend on the scanning mode, photodetector integration time, and number of samples taken. Measurement at one position is expected to take 1 ms.

A typical sensor mast assembly can rotate a full $360^{\circ}$ in azimuth, but has a limited elevation range $\left.{ }^{[30}\right]$. From approximately $2 \mathrm{~m}$ off the ground, SSSI has a resolution of $1 \mathrm{~cm}$ at $10 \mathrm{~m}$, assuming a $1 \mathrm{mrad}$ pointing accuracy. When scanning the full $360^{\circ}$ in the azimuth with $1 \mathrm{mrad}$ steps, the instrument will sample approximately 6300 pixels per circular scan. 840 circular scans are required to cover an area around the rover from $-60^{\circ}$ below level to $10 \mathrm{~m}$ from the base of the mast on level ground. The amount of data generated depends on the scanning mode. The amount of raw data required for each image is 16-bits per wavelength per pixel. Thus, the above full azimuth $10 \mathrm{~m}$ scan would require $233 \mathrm{MB}$ (at 2 bytes/pixel/wavelength) to store raw wavelength data in an uncompressed format. Substances found in the BEST library can be represented with a 16-bit number. Using the 16-bit substance value for each recognized substance would reduce the above data requirement to $10.6 \mathrm{MB}$ assuming all pixels are found in the BEST database. Both raw and substance data will likely compress very well assuming many adjacent pixels will contain the same substance as their neighbors. Redundancy removal will play an important role in reducing the storage and transmission requirements for both raw, unprocessed wavelength data and classified sample data. Classifying samples with the BEST algorithm can be thought of as reducing the size of the 24-column 16-bit raw wavelength data with a 24-to-1 compression ratio to get the single 16-bit substance identifier. Classified samples are expected to be the primary data transferred from SSSI to Earth, though raw wavelength data will be stored temporarily and transferred upón request. When communication bandwidth between Earth and a rover is at a premium, SSSI can continue to operate, transmitting data only when specific substances are found, reducing the communication requirement to close to zero when no interesting substances are present. 


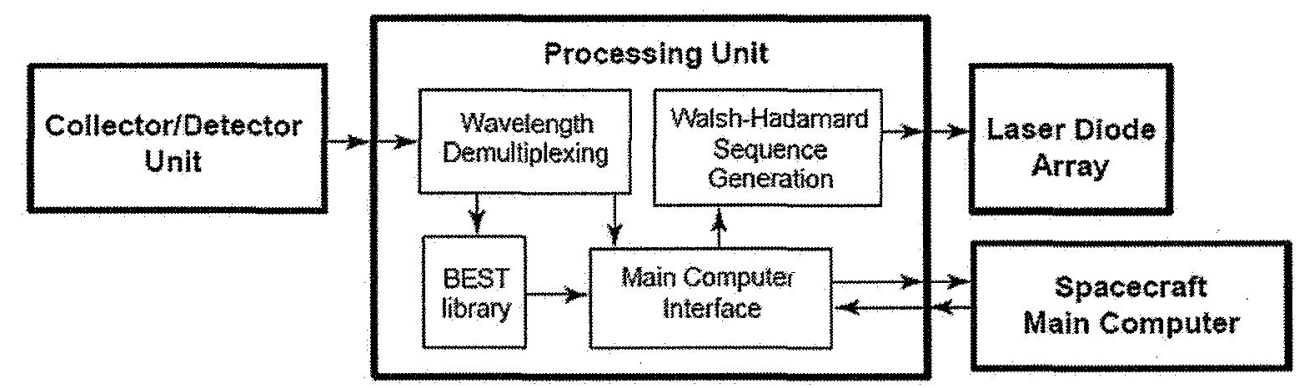

(a) Block diagram of SEARCH

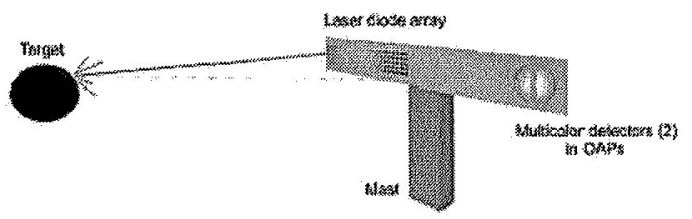

(b) Laser diode anray and collectoridetector unit contiguration

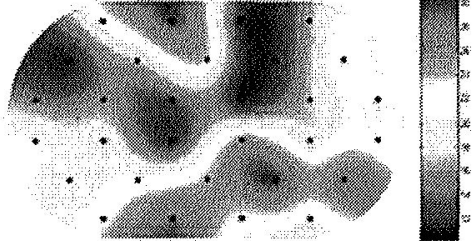

(c) Samoling gratu arer target area

Figure 2. SSSI is composed of (a) the Laser Diode Array, the Collector Detector Unit, and the Processing Unit. The SSSI laser diode array (b) illuminates a target sample with an orthogonal Hadamard pulse sequence. Raster mapping at the black points (c) builds an image indicating what substances are present and their concentrations.

Virtually every organic compound has a near-IR spectrum that can be measured. Near-IR spectra consist of overtones and combinations of fundamental mid-IR bands, giving near- IR spectra a powerful ability to identify organic compounds while still permitting some penetration of light into samples.

In CRISP, the pulse sequences are encoded (using duty cycle) to deliver different amounts of light at each wavelength, such that the total amount of light collected at the detector is linearly related to the analyte concentration of interest. CRISP is a system for generating modulation sequences (or frequencies) such that, using a single detector, the total detector signal integrated over all pulse sequences or modulation frequencies is directly proportional to a specific target analyte concentration. CRISP can be deployed in optical spectrometry using pulses of light as shown in Fig. 3.

In Fig. 3, a Hadamard code table and a CRISP OPR (orthogonal pseudorandom) code table are stored in the instrument. By integrating the detector signal for one pulse sequence length (one frame), CRISP can be implemented with simple 0 or 1 emission pulses. The duty cycle of the sequences is used to control the amount of light delivered at each wavelength. Unlike Hadamard encoding, which imposes several restrictions on the number of pulses in a frame, the length of CRISP OPR codes is set primarily by the number of bits of intensity resolution required. The emitter array's energy consumption depends on the duty cycle of each diode and the sequence length. In addition to reducing duty cycle, it may be possible to use a Shortened CRISP (SCRISP) sequence, depending on the substances in the SSSI biogeochemical library. For example, a Hadamard sequence for the library shown in Figure 3 requires at least four pulses, one for each wavelength. SSSI would use BEST to determine to which substance a given sample is closest. Assuming each diode uses power $P_{d}$ when it is on, the total energy would be:

(4 diodes)(50\% duty cycle)Pd (4 steps)(tstep seconds per step) $=8$ Pdtstep.

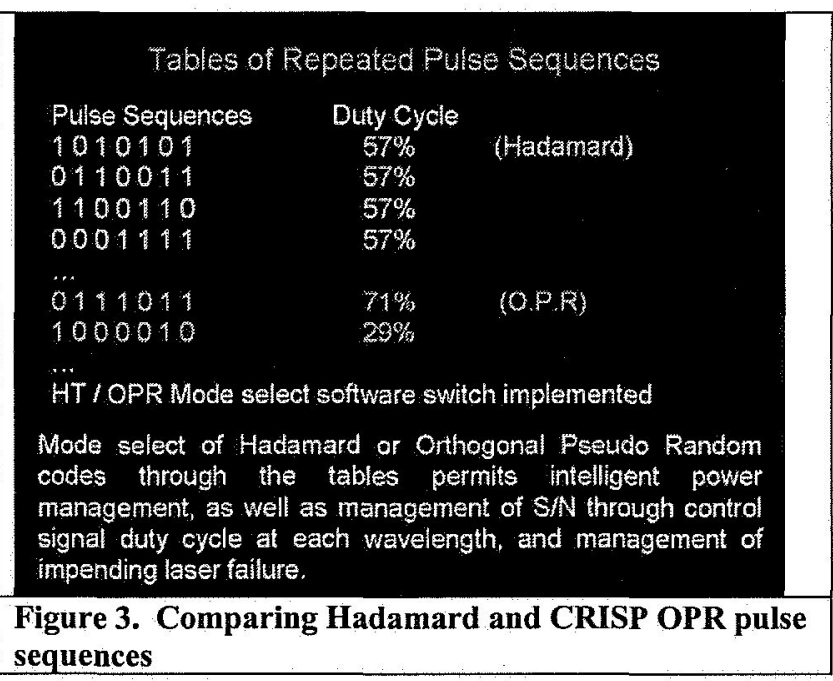

SCRISP can rule out the presence any single substance in a library containing substances with spectra shown in Fig. 4 using a sequence with only two entries. In the figure, substance $A$ is the only substance with a low reflectance at wavelength 1 and a high reflectance at wavelength 2 . The CRISP sequence to detect the presence of substance A could use a $100 \%$ duty cycle for wavelength 1 and a $50 \%$ duty cycle for wavelength 2 . Substance $B$ or C's response read 
from the sensor to the sequence will be approximately twice A's. The total energy consumed to measure a point would be:

( 2 diodes) ( $75 \%$ duty cycle) $P_{d}$ ( 2 steps)(tstep seconds per step $)=3 \mathrm{P}_{\mathrm{d}}$ tstep.

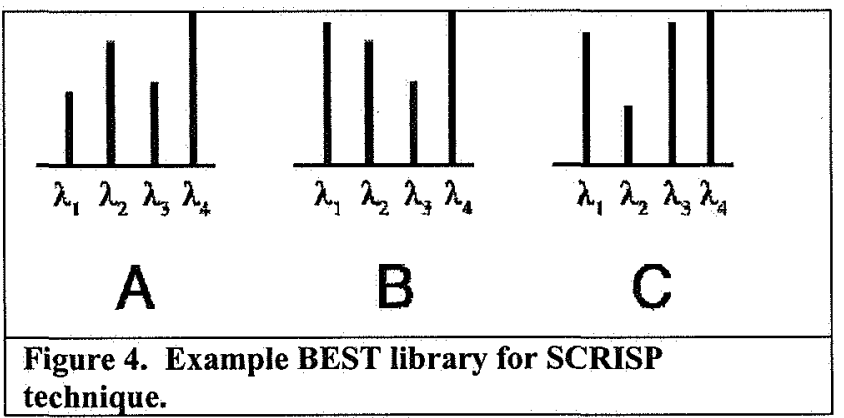

Reducing the number of diodes also reduces the sequence length, yielding a quadratic improvement in total energy used, and linear speedup in scanning. A full-length CRISP sequence is needed to disambiguate any points that match the SCRISP sequence. Though the shortened sequence quickly rules out the presence of any other substance in the library, a substance not in the library with the same spectra response as $\mathrm{A}$ for wavelength 1 and wavelength 2 , but different response for wavelength 3 and wavelength 4 may be present. If any samples in a scan match the expected response for the desired substance, a full-length sequence can be used on the points that match. When the substance being sought is rare, however, the short sequence will be used most of the time, leading to lower average energy consumption and shorter average scanning times. Making the choice between full-length and SCRISP sequences depends on the power consumed by the diodes, the difficulty of distinguishing between a target substance and other substances in the library, and the frequency with which the substance being sought appears.

Energy consumed by the processing unit is a smaller, but still significant contribution to overall power consumption. In SSSI with a Hadamard table, the processing unit demultiplexes each sample point and performs BEST classification on each point.

\section{BEST Sample Classification}

BEST is the analytical basis of SSSI, and the foundation for the biogeochemical library. Spectra recorded at 24 wavelengths are represented as single points in a 24dimensional hyperspace.

A sample spectrum is compared to each substance in a geochemical library based on its distance, measured in standard deviation (SD) units, from the known substances. A sample within 3 SD units of a substance is considered to be composed of the matching substance. Any substance more than 3 SD units away from a known substance is considered an unknown substance.

The BEST algorithm can be suitably approximated using multiple linear regression (MLR) to substantially reduce computational requirements. With the MLR method, the BEST classification algorithm can be performed in situ, allowing a rover to classify large areas, only notifying ground control when an interesting substance is found. When a substance unknown to the BEST library is found, the rover can sample nearby points with similar spectra to create a new library entry for the new substance. Scientists on Earth can determine the type of substance present either by further analyzing raw spectra of the substance provided by SSSI or by using data from the rover's other instruments. Large image regions of spectral data that represent the same substance can be described by a single substance identifier.

\section{Biosignatures and Life-Related Clues}

SSSI can detect and identify surface and endolithic biofilms and soil crusts (caused by photosynthetic Cyanobacteria capable of living under extreme conditions) ${ }^{[31]}$. The instrument can gather chemical data to identify potential habitats. It is suitably small and light to mount on the mast of a stand-alone lander or on that of a rover. SSSI can scan ranges of up to 10 meters. Using a laser-diode array, photodetectors, and on-board processing, SSSI combines innovative spectroscopic integrated sensing and processing with a hyperspace data-analysis algorithm ${ }^{[32]}$. Ultraviolet (UV), visible, and near-infrared laser diodes illuminate target points using a precomputed sequence, and a photodetector records the amount of reflected light from each point illuminated. The resulting reflectance data are processed to separate the contribution of each wavelength of light. The data collected by SSSI are classified and identified by comparison with an extensive data bank library of responses from selected terrestrial materials assembled prior to launch. SSSI spectrometry can also investigate the presence and physical state of water. In addition to its own investigations, SSSI's camera capability would enable it to select optimum targets for itself and for the TWEELs. If the mission contains a rover, SSSI would select areas of interest for application of the full suite of analysis systems, eliminating the need for navcams or other camera systems, substantially reducing the size and cost of the instrument package.

SSSI's laser diode array can obtain profiles of a wide variety of organisms. A sufficient number of discrete reflections at different wavelengths from a target provide a unique profile. In addition, amino acids, carbohydrates, polycyclic aromatic hydrocarbons (PAHS), and more complex organic compounds can be identified.

\section{Presence and Physical State of Water}

The SSSI can detect water in liquid or solid phase, or as hydrates, enabling a rover to follow the water in its search 
for life. The presence, temperature and physical state of water can be determined from near-infrared spectra. Diodes at 1500,1610 , and $1650 \mathrm{~nm}$ would permit such measurement over the range from $20-270^{\circ} \mathrm{K}$. The presence of frost or ice is easily detected, along with its temperature (which changes the hydrogen bond strength) ${ }^{[33]}$. Despite laboratory simulations that show liquid water existing under Martian conditions, various interpretations of the triple point of water and environmental factors affecting it leave the subject in doubt ${ }^{[34],[35]}$. Changes in the near-infrared spectrum of materials have also been used to determine bound and unbound water, particularly in the pharmaceutical industry ${ }^{[36]}$. Unbound water and bound water have been quantified using the spectral region from 1822-1948 nm. Bound water has lower energy stretches (approx. $1930 \mathrm{~nm}$ ) than unbound water (approx. $1910 \mathrm{~nm}$ ). As a result, two diodes are sufficient for this determination in the SSSI. The relationship between water molecules and the strength of the hydrogen bonding between them forms the basis for such determinations ${ }^{[37]}$. Transmission spectra were measured between 1.0 and 2.7 microns for monocrystalline samples of hexagonal water ice at temperatures between 20 and $270 \mathrm{~K}^{[38]}$. Samples were crystallized from liquid water within closed cells, with thicknesses ranging from 100 microns to $1.0 \mathrm{~cm}$. The absorption spectrum of ice changes with temperature in several ways. With higher temperature, the shapes of absorption bands become more smoothed, the strengths of some absorption bands decrease, the absorption in continuum wavelengths increases, and the band centers of some absorption bands shift to shorter wavelengths.

\section{Detection of Chirality}

Near-infrared spectra consist of overtones and combinations of fundamental mid-infrared bands, giving near-infrared spectra a powerful ability to identify organic compounds while still permitting some penetration of light into samples ${ }^{39}$. Near-IR spectrometry has been used successfully to analyze mixtures of optically active compounds including sugars, amino acids, carbohydrates, yeastolate, proteins, and cell debris ${ }^{[40]}$. Enantiomeric purity has even been assayed via near-infrared reflectance spectroscopy ${ }^{[1]}$, suggesting that the SSSI might be a valuable complement to microbial chiral LR metabolic experiments.

\section{Detection of Gas Traces}

Infrared laser diodes can also measure gases in small cells at ppm or even ppb levels such as might accumulate in an LR experiment (see Table 3). Methane does not have a discrete absorption window like other gases. As a result, multiple infrared diodes and chemometric regression methods are required to simultaneously measure water, carbon monoxide carbon dioxide, and methane. Carbon dioxide, water, and methane gases can be determined using three diodes between 2500 and $3300 \mathrm{~nm}$ (see Figure 5). A water sensor could easily fit within $20 \mathrm{~cm}^{3}$.
Table 3. Overlap of Absorption Bands of Gases (Micrometers)

\begin{tabular}{|l|c|}
\hline \multicolumn{1}{|c|}{ Gases } & Absorption Bands \\
\hline $\mathrm{H}_{2} \mathrm{O}$ & $0.5-2.0$ \\
\hline $\mathrm{H}_{2} \mathrm{O}, \mathrm{CO}$, and $\mathrm{CO}_{2}$ & $2.0-3.0$ \\
\hline $\mathrm{H}_{2} \mathrm{O}$ and $\mathrm{CH}_{4}$ & $3.0-4.0$ \\
\hline $\mathrm{CO}$ and $\mathrm{CO}_{2}$ & $4.0-5.0$ \\
\hline $\mathrm{H}_{2} \mathrm{O}$ & $5.0-7.0$ \\
\hline $\mathrm{CH}_{4}$ & $7.0-8.0$ \\
\hline $\mathrm{CO}_{2}$ and $\mathrm{CH}_{4}$ & $13.7-14.7$ \\
\hline $\mathrm{CO}_{2}$ & $14.7-16.5$ \\
\hline 110$]$ & \\
\hline
\end{tabular}

The Martian atmosphere is $95.32 \%$ carbon dioxide, $0.07 \%$ carbon monoxide, and $0.03 \%$ water vapor. The methane concentration is about 10.5 parts per billion on Mars. (In contrast, the current atmospheric concentration of methane is about $1.7 \mathrm{ppm}$ by volume on Earth.) The infrared detection limit is about $10 \mathrm{ppb}$ for water in nitrogen gas using a conventional interferometer.

\section{5. $p H$ Determination}

Near-infrared spectra are a sensitive probe of $\mathrm{pH}$. Standard error of prediction (SEP) values of $0.034 \mathrm{pH}$ units have been obtained in this manner ${ }^{[42]}$. Thus, the SSSI could also be used to measure $\mathrm{pH}$ in the soil.

\section{INSTRUMENTATION}

The two main components of this instrument assembly are the TWEELs in their canister and the SSSI. During terminal heat sterilization, launch, flight and landing, the instrument package is hermetically sealed in its canister. After landing, the signal to deploy is given. This releases a détente that allows the spring-loaded telescoped mast to puncture through the sterile plenum comprising the lid of the canister. The mast rises approximately one-half meter above the ground. The TWEEL is shown in Figure 6. The deployed array is seen is Figure 7. The TWEEL pod sits on top of the mast, with the SSSI array beneath it.. The mast is free to rotate into the wind. A verticality sensor actuates and directs the mast to aim the TWEELs and SSSI at the desired (programmed or commanded) angle with the vertical. The SSSI camera surveys the upwind area and permits target selection. The TWEEL squibs are fired at selected targets individually, with time allowed to ascertain whether a TWEEL has landed successfully and begun obtaining data prior to firing the next. Using the knee joint in the mast, successive TWEELs can be fired at different elevations to impact different areas. If on a rover, the SSSI will have greater ability to select targets, and the TWEELs may be 


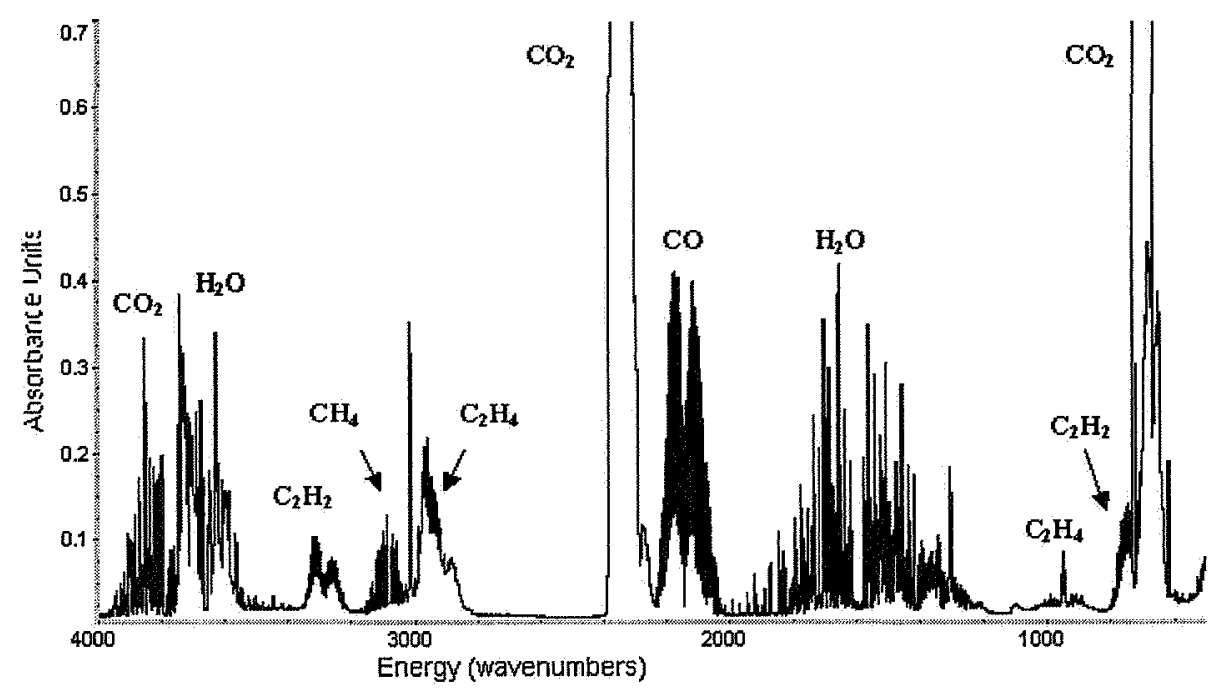

Figure 5. Infrared Spectra of Gases

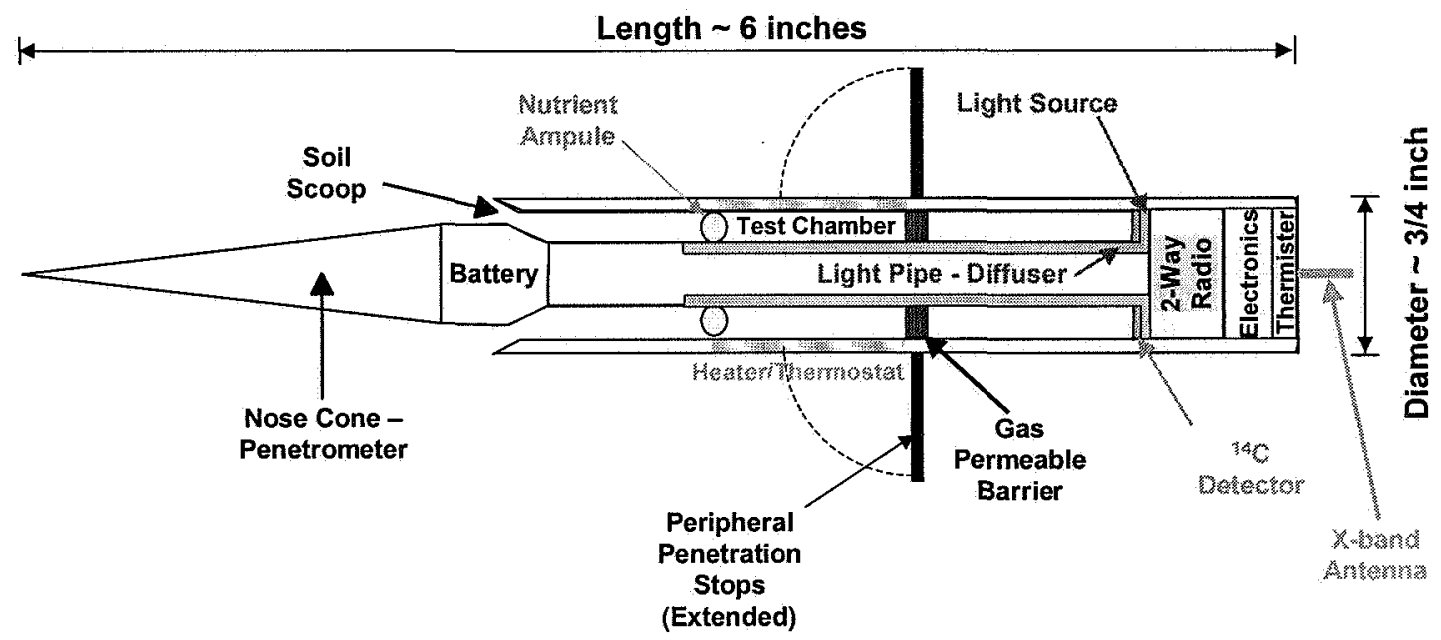

Note Sterilized canister contains multiple probes that are ejected away from spacecraft after landing.

Figure 6. Twin Wireless Extraterrestrial Experiment for Life (TWEEL) 


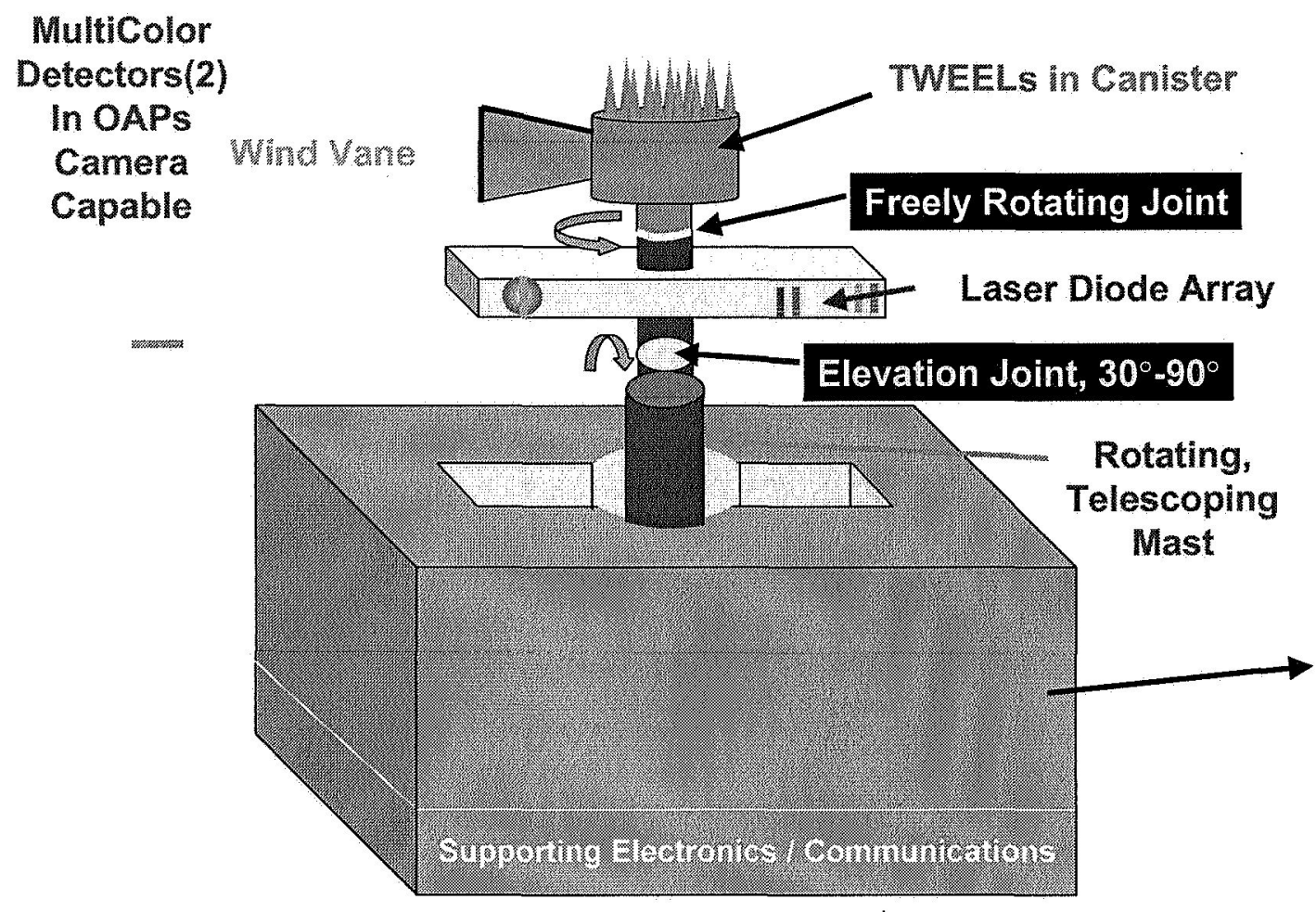

Figure 7. Deployed TWEEL/SSSI

fired from different locations, providing the rover always travels upwind until all TWEELs are deployed. In either case, static or rover, the SSSI functioning as a camera will provide guidance to target areas of interest as determined by the SSSI.

It is planned to use a TWEEL for each of the seven nutrients supplied by the Viking LR as a benchmark for the TWEEL experiment, and to seek to confirm the Viking findings. The principal difference will be that the alanine and lactate will each be supplied in the forms of their respective optical isomers. L- and D- cysteine will constitute two additional TWEELs. Depending on final weight determinations for the instrument, and on their selection, several additional nutrients may be incorporated into TWEELs, for a total number of 12 to 16 .

After the TWEEL firings, the SSSI will identify specific targets for its own analyses. The targets will be illuminated by the laser diodes, the reflections acquired and instantly fed to the on-board computer library for identification. They can also be relayed to the more extensive, Earth-based SSSI data library.

\section{STERILITY}

Among stringent COSPAR requirements for any spacecraft carrying a life detection experiment is terminal sterilization of the entire spacecraft. The rationale is to protect Mars from terrestrial microbial contamination, and to protect the integrity of the experiment by precluding a false positive result through detection of microorganisms that hitch-hiked a ride from Earth. In the case of Viking, its sterilization consumed 25 percent of the entire project budget. The approach here is to make the sterilization affordable by dedication of the mission to the proposed experiments and instrument package only. The spacecraft will then be small enough to permit its economical sterilization. However, should the package be selected for a multiple purpose mission, an alternate method of economic sterilization has been developed $\mathrm{d}^{[43]}$ whereby only the proposed experiment is sterilized. As described above, the method consists of sealing the entire instrument package inside a canister that can be simply attached to a designated fixture on a lander or rover, depending on the nature of the mission. Prior to launch, the entire canister is heat sterilized. It is then attached to the lander or rover receptacle fixture. Upon activation of the experiment after landing, release of the detent permits the mast to extend, thereby puncturing through the sterile plenum top of the canister. Free to rotate into the wind, the assembly assures that TWEELs will be launched upwind, approximately 100 meters away from any terrestrial contamination that may have been deposited by the spacecraft. This precautionary measure will insure integrity of the experiment and, also, prevent planetary contamination from these experiments. 


\section{Preliminary Size, Weight ANd Power SPECIFICATIONS}

Best estimates at the present stage of development:

$$
\begin{aligned}
& \text { Size of canister } \sim 0.06 \mathrm{~m}^{3} \\
& \text { Total weight of package } \sim 3 \mathrm{~kg} \\
& \text { Power } \\
& \text { Per TWEEL } \sim 200 \mathrm{~mW} \text { (supplied by } \\
& 2 \text { lithium hydride batteries) } \\
& \text { x } 16 \text { TWEELs }=\sim 3.2 \mathrm{~W} \\
& \text { SSSI } \sim 100 \mathrm{~mW} \\
& \text { Radio/computer/support } \sim 500 \mathrm{~mW} \\
& \text { Total power } \sim 4.1 \mathrm{~W}
\end{aligned}
$$

\section{INCREASED PROSPECTS THAT THE VIKING LIFE DETECTION EXPERIMENT SUCCEEDED AND ITS PORTENT}

The question of whether life is restricted to planet Earth or more widely distributed in the Cosmos is truly one of the most profound questions to be addressed by modern science.

During the 40 years that have transpired since the Viking Landers conducted their search for evidence of life on Mars, much new information has been gained about microbial extremophiles and water in the polar caps and permafrost of Mars. This knowledge greatly increases the prospects that the Viking life detection experiments actually succeeded. It is now known that there exists a great biodiversity of microbial extremophiles that live in the cryopegs (sodiumchloride water brines in the permafrost) of the Kolyma lowlands of Northeast Siberia ${ }^{[4]}$. The cryopegs found in the polar regions of Earth are permanently cryotic overcooled water-brine lenses in marine sediments. They are prevented from freezing due to the extremely high salinity of the pore water. Cryopegs remain perpetually liquid under extremely frigid conditions and therefore they represent the best astrobiological analogs as proxies for microbial communities that might inhabit the Martian permafrost $^{[45],[46]}$. Over significant geological time periods (hundreds of thousands to millions of years) these ecosystems have never been exposed to temperatures above $0^{\circ} \mathrm{C}$. Their content of the dissolved solids can be so high that cryopegs can remain in liquid state at -10 to $-15{ }^{\circ} \mathrm{C}$ or lower. Water-brine samples from $20 \mathrm{~m}$ thick sodium chloride marine horizon of a 100,000-120,000 year old Pleistocene cryopeg in NE Siberia were found to contain astonishingly high $\left(10^{6}-10^{7}\right.$ cells $\left./ \mathrm{ml}\right)$ cell counts. This community exhibited a very great microbial diversity. It was comprised of aerobic cocci and coccobacilli, mycelial fungi, and yeast and anaerobic bacteria and archaea, halophilic sulfate reducers, heterotrophs, acetogens and methanogens. The incorporation of ${ }^{14} \mathrm{C}$ from labeled glucose provided evidence that some of these microorganisms were capable of metabolic activity at temperatures as low as $-15^{\circ} \mathrm{C}$. It is now well known that viable microorganisms can be cryopreserved for geological time periods while frozen in the deep layers the Central Antarctic ice sheet ${ }^{[4]}$. Furthermore, the novel species of bacterium, Carnobacterium plesitocenium, which had remained frozen in a thermokarst pond of the Alaskan permafrost for 32,000 years, exhibited motility immediately after the ice samples were allowed to thaw on a sterile slide under the microscope $\left.{ }^{[48}\right]$. The psychrotolerant microorganism Trichococcus patagoniensis has been found to exhibit not just metabolic activity, but replication and growth at $-5^{\circ} \mathrm{C}$. $[49\}$.

These recent discoveries clearly indicate that cryotic microorganisms in the upper layers of the Martian regolith could very well have initiated both metabolic activity and replication when the Mars soil samples were exposed to liquid water and the ${ }^{14} \mathrm{C}$ labeled nutrients during the 1976 Viking Mission. Hence, the LR life detection experiment may very well have yielded valid detection of microbial life on Mars, which was erroneously attributed by many to a chemical rather than a biological phenomenon.

The use of the phenomena of chirality, photosynthesis, and circadian rhythms to differentiate between chemistry and biology gives the TWEEL/SSSI instrument package the ability to differentiate between not only chemistry and biology but also between Earth-like biological systems and a biology that might utilizes sugars and amino acids of alternate chirality.

The verification that life exists on Mars today is would represent an observation of the most profound significance. One of the most basic scientific and philosophical questions of all time has been "Are we Alone?" The TWEEL/SSSI instrument complex makes it possible to go beyond the basic question "Does life exist only on planet Earth or is it a Cosmic Imperative?" The TWEEL/SSSI results could provide crucial information relevant to the origin of life on Earth, and help delineate the origin, evolution and extent of the biosphere. The photosynthesis and circadian rhythm components could provide clues to fundamental aspects of the metabolism and life processes of the organisms. Should it be determined that life exists on Mars, and that it utilizes a biochemistry based on the same enantiomers used by life on Earth, this would suggest that life may have sprung from a single origin and been distributed to other bodies of the cosmos by a panspermia-type transfer mechanism via impact ejection phenomena or propagation by comets, asteroids or interstellar dust. However, if life on Mars should be found to construct proteins, DNA and RNA by the use L-sugars and D-amino acids, it would strongly imply that at least two separate and distinct events were responsible for the different life forms. The validation that life exists today on Mars, or anywhere else in the solar system, would truly revolutionize our understanding of the nature of the universe, and open our thinking to the possibility of a biologic imperative. 


\section{ACKNOWLEDGEMENTS}

The authors express their deep thanks to Mrs. Kathy Brailer, Spherix Inc., for her excellent and essential help in assembling this manuscript.

\section{REFERENCES}

[1]. Levin, G.V. and P.A. Straat, "Viking Labeled Release Biology Experiment: Interim Results," Science, 194, 1322-1329, December 1976.

[2]. Levin and Straat, NAS talk proceedings, 1986.

[3]. Feldman, W.C. et al., "Global Distribution of Neutrons from Mars: Results from Mars Odyssey," Science 297, 75-78, 2002.

[4]. Mitrofanov, I. et al., "Maps of Subsurface Hydrogen from the High-Energy Neutron Detector, Mars Odyssey," Science 297, 78-81, 2002.

[5]. Boynton, W.V. et al., "Distribution of Hydrogen in the Near-Surface of Mars: Evidence for Subsurface Ice Deposits," Science 297, 81-85, 2002.

[6]. Hecht, M., "Aqueous environments on contemporary Mars," Instruments, Methods, and Missions for Astrobiology IV, Proceedings of SPIE, 4495, 69-80, 2001.

[7]. "Mars rovers explore hints of salty water: Water one of several theories proposed," by Robert Roy Britt, SPACE.com, February 19, 2004, htp:/www.cnn.com/2004/TECH/space/02/19/shc.mar s. search/.

[8]. "Earthlings Discover There Might Be Life On Mars After All," S. Begley, Wall St. Jour., Oct. 24, 2006.

[9]. Krasnopolsky, V.A., J. P. Maillard, and T. C. Owen, "Detection of Methane in the Martian Atmosphere: Evidence for Life," European Geosciences Union, 1st General Assembly, Nice, France, April 25-30, 2004.

[10]. Howe, L., "Formaldehyde, Ammonia, and Benzene Molecules on Mars? Would Probably Mean Life," www.earthfiles.com/news/printerfriendly.cfm?id=707).

[11]. S. Ryan et al., "Mauna Loa volcano is not a methane source: Implications for Mars," Geophys. Res. Letter, 33, 112301 doi: 10.1029GLO26223, 2006.

[12]. Thomas, D.N. and Dieckmann, G.S., "Antarctic sea ice - a habitat for extremophiles," Science 295, 641644, 2002.

[13]. E.J. Carpenter, S. Lin, and D.G. Capone, "Bacterial Activity in South Pole Snow," Applied and Environmental Microbiology 66, 10, 4514-4517, 2000.

[14]. R.H. Vreeland, W.D. Rosenweig, and D.W. Powers, "Isolation of a 250 million-year-old halotolerant bacterium from a primary salt crystal," Nature 407, 897-900, 2000.

[15]. E.M. Rivkina, E.I. Friedmann, C.P. McKay, and D.A. Gilichinsky, "Metabolic Activity of Permafrost Bacteria Below the Freezing Point," Applied and Environmental Microbiology 66, 8, 3230-3233, 2000.

[16]. M.L. Skidmore, J.M. Foght, M.J. Sharp, "Microbial Life Beneath a High Arctic Glacier," Applied and Environmental Microbiology 66, 8, 3214-3220, 2000.
[17]. Levin, G.V. and P.A. Straat, "Antarctic Soil No. 726 and Implications for the Viking Labeled Release Experiment," J. Theor. Biol., 91, 41-45, 1981.

[18]. Fisk, M., "Life in Tiny Tunnels?" as reported in Astrobiology online, Sunday, March $26 @ 00: 46: 03$, 2006.

[19]. Levin, G., "Scientific Logic for Life on Mars," Instruments, Methods, and Missions for Astrobiology, SPIE Proceedings, 4495, 81-88, 2001.

[20]. Op cit 3 .

[21]. Op cit 4.

[22]. Op cit 5.

[23]. Op cit 6.

[24]. Op cit 7.

[25]. Paepe, R, RB Hoover and E Van Overloop, "Patterned Ground as Evidence of Water on Mars," in Permafrost Response on Economic Development, Environmental Security and Natural Resource Potential, NATO-ARW, held in Novosibirsk, Siberia, Nov. 12-16, 1998, Roland Paepe, Ed., Klewer Publishing, New York, 581-588, 2001.

[26]. Weinbaum, S.G., A Martian Odyssey and Other Science Fiction Tales, Hyperion Press, June 1974.

[27]. Toulmin, P. III et al., "Geochemical and Mineralogical Interpretation of the Viking Inorganic Chemical Results," J. Geophys. Res. 82, 4625, 1977.

[28]. Miller, JD, PA Straat and GV Levin, "Periodic Analysis of the Viking Lander Labeled Release Experiment," Instruments, Methods, and Missions for Astrobiology, SPIE Proceedings, 4495, 96-107, July 2001.

[29]. H. E. B. Da Silva, C. Pasquini, Dual-Beam NearInfrared Hadamard Spectrophotometer Appl. Spectrosc. 55 (6), 715-721, 2001.

[30]. W. Dieter, R.A. Lodder, J.E. Lumpp, Jr., "Scanning for Extinct Astrobiological Residues and Current Habitats (SEARCH) Using Integrated Computational Imaging," Aerospace, 2005 IEEE Conference, March 2005, 234- 245, ISBN: 0-7803-8870-4, Digital Object Identifier: 10.1109/AERO.2005.1559317.

[31]. F. Garcia-Pichel, S.L. Johnson, D. Youngkin, and J. Belnap, "Small-scale vertical distribution of bacterial biomass and diversity in biological soil crusts from arid lands in the Colorado Plateau," Microbial Ecol $46: 312-321,2003$.

[32]. W.R. Dieter, R.A. Lodder, J.E. Lumpp, Jr., Aerospace, 2005 IEEE Conference, 5-12 March 2005, 234- 245, DOI: 10.1109/AERO.2005.1559317.

[33]. F. Garcia-Pichel and R. W. Castenholz, "Occurrence of UV-Absorbing, Mycosporine-Like Compounds among Cyanobacterial Isolates and an Estimate of Their Screening Capacity," Appl. Environ. Microbiol., Vol. 59, No. 1, 163-169, Jan 1993.

[34]. S.M. Clifford and T.J. Parker, "The Evolution of the Martian Hydrosphere: Implications for the Fate of a Primordial Ocean and the Current State of the Northern Plains," Icarus 154, 40-79, 2001. 
[35]. "Opportunity rover finds strong evidence Meridiani Planum was wet," NASA/Jet Propulsion Laboratory press release, March 2, 2004, Available at http://marsrovers.jpl.nasa.gov/ newsroom/pressreleases/20040302a.html.

[36]. K.R. Morris, S.L. Nail, G.E. Peck, S.R. Byrn, U.J. Griesser, "Advances in pharmaceutical materials and processing," Pharm. Sci. Technol. Today, 1998, Vol. 1, No. 6, 235-245.

[37]. M. Falk and T.A. Ford, "Infrared Spectrum and Structure of Liquid Water," Can. J. Chem./Rev. can. chim. 44(14): 1699-1707 (1966).

[38]. W.M. Grundy and B. Schmitt, "The TemperatureDependent Near-Infrared Absorption Spectrum of Hexagonal H2O Ice," J. Geophys. Res. 1998, 103, 25,809-25.

[39]. R.J. Dempsey, D.G. Davis, R.G. Buice, Jr., R.A. Lodder, "Biological and Medical Applications of Near-Infrared Spectrometry;" Appl. Spectrosc., 50(2), 18A-34A, 1996.

[40]. M.R. Riley, M. Rhiel, X. Zhou, M.A. Arnold, D.W. Murhammer, "Simultaneous measurement of glucose and glutamine in insect cell culture media by near infrared spectroscopy," Biotechnology and Bioengineering, 55(1), 11 - 15, 26 Mar 2000.

[41]. B.R. Buchanan, E.W. Ciurczak, A.Q. Grunke, D.E. Honigs, "Determination of enantiometric purity of valine via near-infrared reflectance spectroscopy," Spectroscopy (Duluth, MN, United States) (1988), 3(5), 54-6.

[42]. M.K. Alam, J.E. Franke, M.R. Rohrscheib, D. Nunez, V. Abate, J.D. Maynard, G.J. Kemeny, "Hemoglobin correction for near-infrared $\mathrm{pH}$ determination in lysed blood solutions," Appl Spectrosc. 2003 Sep;57(9):1093-9.

[43]. Levin, G.V., J.D. Miller, P.A. Straat, and R.B. Hoover, "A Sterile Robotic Mars Soil Analyzer," Instruments, Methods, and Missions for Astrobiology, SPIE Proceedings, 4859, 78-86, August 2002.

[44]. D. Gilichinsky, E. Rivkina, V. Shcherbakova, K. Laurinavichuis, J. Tiedje, "Supercooled water brines within permafrost-an unknown ecological niche for microorganisms: a model for astrobiology," Astrobiology, 2003, Summer;3(2): 331-341.

[45]. R.L. Mancinelli, T.F. Fahlen, R. Landheim and M.R. Klovstad, "Brines and evaporites: analogs for Martian life," Advances in Space Research, 33, 2004, 1244 1246.

[46]. D. Gilichinsky, E. Rivkina, C. Bakermans, V. Shcherbakova, L. Petrovskaya, S. Ozerskaya, N. Ivanushkina, G. Kochkina, K. Laurinavichuis, S. Pecheritsina, R. Fattakhova and J.M. Tiedje, "Biodiversity of cryopegs in permafrost," FEMS Microbiology Ecology, 2005 June; 53(1), 117-128.

[47]. S.S. Abyzov, R.B. Hoover, S. Imura, I.N. Mitskevich, T. Naganuma, M.N. Poglazova, and M.V. Ivanov, "Comparative Results of Using Different Methods for Discovery of Microorganisms in Very Ancient Layers of the Central Antarctic Glacier above Lake Vostok." Advances in Space Research, COSPAR, 2004, 33, 1222-1230, (2004).

[48]. E.V. Pikuta, R.B. Hoover, D. Marsic, A..K. Bej, J. Tang, and P. Krader, "Carnobacterium pleistocaenium sp. nov., a novel psychrotolerant, facultative anaerobe isolated from Fox Tunnel permafrost, Alaska." Int $J$ Syst Evol Microbiol, 55, 2005, 473-478.

[49]. E.V. Pikuta, R.B. Hoover, A.K. Bej, D. Marsic, W.B. Whitman, P.E. Krader, and J. Tang, "Trichococcus patagoniensis sp. nov., a facultative anaerobe that grows at $-5{ }^{\circ} \mathrm{C}$, isolated from penguin guano in Chilean Patagonia," Int J Syst Evol Microbiol, 56, 2006, 2055-2062. 